\title{
Interleukin-6-induced epithelial-mesenchymal transition through signal transducer and activator of transcription 3 in human cervical carcinoma
}

\author{
JIN-WEI MIAO ${ }^{1}$, LI-JIANG LIU ${ }^{2}$ and JIE HUANG ${ }^{2}$ \\ ${ }^{1}$ Department of Gynecologic Oncology, Beijing Obstetrics and Gynecology Hospital, Capital Medical University, \\ Beijing 100026; ${ }^{2}$ Department of Pathology and Pathophysiology, School of Medicine, \\ Jianghan University, Wuhan 430056, P.R. China
}

Received January 26, 2014; Accepted March 24, 2014

DOI: $10.3892 /$ ijo.2014.2422

\begin{abstract}
Epithelial-mesenchymal transition (EMT) is an important process in the invasion and metastasis of human cervical carcinoma. The pro-inflammatory cytokine interleukin-6 (IL-6) has been shown as an EMT inducer in multiple carcinomas. However, whether the EMT program can be induced by IL-6 and the mechanisms underlying the IL-6-induced EMT in human cervical carcinoma remain to be determined. In this study, we show that IL-6 receptor (IL-6R) and signal transducer and activator of transcription 3 (Stat3) were highly expressed in human cervical squamous cell carcinoma (CSCC) tissues, and the expression of EMT markers was reversed in well-differentiated and poorly-differentiated human CSCC. Additional experiments showed that IL-6 exposure in cervical carcinoma cell lines induced IL-6R and Stat 3 expression, markedly promoted cell growth, and altered cell morphology. The treatment of cervical carcinoma cell lines with IL-6 resulted in downregulation of E-Cadherin and upregulation of Vimentin. Importantly, knockdown of Stat3 significantly reversed the IL-6-induced EMT program, suggesting that Stat3 is necessary for IL-6-induced EMT in the progression of human cervical carcinoma. Moreover, Slug, a member of the Snail family of EMT regulators, was observed to be associated with the expression of Stat3. We concluded that IL-6 plays an important role through Stat 3 in the EMT induction and can be a potential therapeutic target and biomarker for human cervical carcinoma.
\end{abstract}

Correspondence to: Dr Jie Huang, Department of Pathology and Pathophysiology, School of Medicine, Jianghan University, Wuhan 430056, P.R. China

E-mail: huang6149003786@gmail.com

Key words: interleukin-6, signal transducer and activator of transcription 3, epithelial-mesenchymal transition, human cervical carcinoma

\section{Introduction}

Human cervical carcinoma is one of the leading malignancies in women worldwide (1). Approximately $80 \%$ of human cervical carcinoma is squamous cell carcinoma (SCC), while only $20 \%$ of remaining cervical carcinoma is adenocarcinoma (2). Epidemiological and laboratory data have shown that human cervical carcinoma is mainly associated with human papillomavirus (HPV) infections $(3,4)$. The HPV infection and replication in cervical squamous epithelial cells resulted in increasing the risk of developing cervical carcinoma (5). However, accumulated data indicate that only a small number of women infected with HPV finally develop a highly malignant cervical carcinoma, suggesting that other factors are necessary for development of human cervical carcinoma (6).

Interleukin-6 (IL-6) is a central pro-inflammatory cytokine, which has been described as an important factor for the pathogenesis of human cervical carcinoma (7). In addition, IL-6 is highly expressed in invasive cervical carcinoma and is associated to the pathogenesis of HPV-related cervical carcinoma (8). These data support the hypothesis that IL-6 signaling may participate in the development of human cervical carcinoma. Classic IL-6 signaling requires binding of IL-6 to interleukin-6 receptor (IL-6R) and subsequent activation of signal transducer and activator of transcription 3 (Stat3), a downstream protein of IL-6R (9). Stat3 is typically located in the cytoplasm when Stat3 is inactive, then Stat 3 forms homodimers and enters the nucleus binding to DNA-response elements after phosphorylation of Stat3 (10). Stat3 has been demonstrated to play an important role in human cervical carcinoma and its activation by IL- 6 stimulation is connected with enhanced cancer cell growth, survival and immune evasion (11-13).

Epithelial-mesenchymal transition (EMT) was originally described as an essential process for specific developmental stages and embryonic epithelial cells migration in early embryogenesis (14). Studies have also shown that EMT is an important process in many types of human epithelial carcinoma including cervical carcinoma (15-19). During EMT, cancer cells lose their epithelial properties and gain mesenchymal characteristics (20). As an epithelial cell marker, E-Cadherin 
is a cell adhesion molecule and is associated with suppression of cancer cell motility and invasion (21), whereas, the expression of mesenchymal cell marker Vimentin in cancer cells is believed to enhance migration and invasiveness, and is regulated by several transcription factors including Slug in cancer progression $(22,23)$. Several factors such as EGF are known to regulate EMT in human cervical carcinoma (19). However, whether IL-6 plays an important role in EMT induction and the mechanisms underlying the IL-6-induced EMT remain to be determined.

In this study, we investigated the role of IL-6 and its downstream protein Stat 3 in regulating the EMT program in human cervical carcinoma. We show that IL-6R and Stat 3 were highly expressed in human cervical squamous cell carcinoma (CSCC) tissues, and the expression of EMT markers was reversed in well-differentiated and poorly-differentiated human CSCC. Furthermore, IL-6 exposure of cervical carcinoma cell lines induced IL-6R and Stat 3 expression, markedly promoting cell growth, and altered cell morphology. The treatment of cervical carcinoma cells with IL-6 resulted in significant upregulation of Vimentin expression and downregulation of E-Cadherin expression. Knockdown of Stat 3 in cervical carcinoma cells significantly reversed the IL-6-induced EMT program, suggesting that Stat 3 is necessary for IL-6-induced EMT in progression of human cervical carcinoma. Moreover, we show a correlation between Stat 3 and EMT regulatory factor Slug in cervical carcinoma cells. Our findings, therefore, identify a novel mechanism of IL-6-induced EMT program and a potential therapeutic target for human cervical carcinoma.

\section{Materials and methods}

Primary antibodies and reagents. Recombinant human IL-6, human IL- 6 receptor $\alpha$ and $\beta$ antibodies, IL- 6 receptor inhibitor and $\beta$-actin were obtained from Sigma. Stat 3 and p-Stat 3 antibodies were obtained from Cell Signaling Technology. E-Cadherin and p-Slug antibodies were obtained from BD Transduction Laboratories. Ki67 antibody was obtained from Dako. CK8, CK14, CDK4, Cyclin D1 and p63 antibodies were obtained from Santa Cruz Biotechnology, Inc. Vimentin antibody was obtained from Chemicon.

Human normal and cervical squamous cell carcinoma tissues. Human normal and CSCC tissues were obtained from the Department of Pathology and Pathophysiology, School of Medicine, Jianghan University in Wuhan, China, and from Department of Gynecologic Oncology, Beijing Obstetrics and Gynecology Hospital, Capital Medical University in Beijing, China, respectively. The ethics were reviewed and approved by the board of the hospital at Jianghan University and by the board of the hospital at Capital Medical University. Patients with previous radiotherapy or chemotherapy were excluded from this study. All human normal and CSCC tissues were fixed in $10 \%$ formalin until analyzed.

Cell culture. The HeLa cervical cancer cell line (HPV-positive) and the C33A cervical cancer cell line (HPV-negative) were obtained from American Type Culture Collection. Both cell lines were grown in Dulbecco's Modified Eagle's medium (DMEM) (HyClone) supplemented with 10\% fetal bovine serum (FBS) (Gemini Bio-Products) and 1\% penicillin/ streptomycin solution (Thermo Fisher Scientific). Cells were incubated at $37^{\circ} \mathrm{C}$ in a humidified incubator with $5 \% \mathrm{CO}_{2}$ until cells reach $80 \%$ confluence, then cultured in serum-free medium for $24 \mathrm{~h}$. Triplicate wells of cells were incubated for up to $48 \mathrm{~h}$ in the presence of $0-200 \mathrm{ng} / \mathrm{ml} \mathrm{IL}-6$. In some experiments, cells were also treated with $10 \mu \mathrm{M}$ curcumin (an inhibitor of IL-6 receptor). Cells were evaluated for mRNA and/or protein levels of IL-6 receptor, E-Cadherin, Vimentin, p-Slug, p-Stat3 and Stat3.

Tissue immunofluorescence staining. Human normal and CSCC tissues were fixed in $10 \%$ formalin and embedded in paraffin. After deparaffinization, sections were boiled in an antigen unmasking solution for $15 \mathrm{~min}$ and blocked in 5\% non-fat milk in PBS for $30 \mathrm{~min}$ at room temperature. Sections were incubated with primary antibodies (Ki67, 1:200; CDK4, 1:100; Cyclin D1, 1:100; CK8, 1:100; CK14, 1:200; p63, 1:50; E-Cadherin, 1:100; Vimentin, 1:200; IL-6R $\alpha, 1: 100$; IL-6R $\beta$, Stat3, 1:100; and p-Stat $1: 100$ ) overnight at $4^{\circ} \mathrm{C}$, followed by incubation with fluorescent-conjugated secondary antibodies (Molecular Probes) for $1 \mathrm{~h}$ at room temperature in the dark. Mounting medium (Vector Laboratories) was used with 4',6'-diamino-2-phenylindole (DAPI) at $1 \mu \mathrm{g} / \mathrm{ml}$ for $30 \mathrm{~min}$, and the sections were analyzed by fluorescence microscopy with a Carl Zeiss Axioscope (Carl Zeiss).

Immunocytochemical staining. Following treatment with IL-6 $(50 \mathrm{ng} / \mathrm{ml})$ or IL- 6 + curcumin $(10 \mu \mathrm{M})$ for $48 \mathrm{~h}, \mathrm{HeLa}$ and C33A cells were plated on 8-well coverslips and fixed with ice cold methanol for $5 \mathrm{~min}$ at $-20^{\circ} \mathrm{C}$. Cells were washed 3 times with ice-cold PBS, exposed to $0.1 \%$ Triton X-100 in PBS for 5 min, and then washed with $0.1 \%$ Triton-X 100 in PBS. Cells were incubated with primary antibodies diluted in PBS with $3 \%$ bovine serum albumin (BSA) followed by a $1-\mathrm{h}$ incubation at room temperature in $0.1 \%$ Nonidet P-40 in PBS. Coverslips were incubated with a solution containing fluorescent isothiocyanate-conjugated goat anti-mouse IgG (green) (Wako Pure Chemical Industries) and Rhodamine-conjugated goat antirabbit IgG (red) (Sigma) for $1 \mathrm{~h}$ at room temperature. Finally, the coverslips were mounted with 4',6'-diamino-2-phenylindole (DAPI) at $1 \mu \mathrm{g} / \mathrm{ml}$ for $30 \mathrm{~min}$ and analyzed by fluorescence microscopy with a Carl Zeiss Axioscope (Carl Zeiss, Inc.).

RNA extraction and real-time polymerase chain reaction $(P C R)$. Total RNA was extracted using RNA STAT-60 (Tel-Test) according to the manufacturer's protocol. First-strand cDNA was synthesized using $1 \mu \mathrm{g}$ of total RNA in a $20-\mu \mathrm{l}$ final volume by reverse transcription utilizing SuperScript II Reverse Transcriptase (Invitrogen) with oligo-dT(18)-primers (Invitrogen). The real-time PCR reactions were performed using SYBR-Green Master Mix kit according to the manufacturer's instructions (Applied Biosystems). RNA for IL-6, E-Cadherin, Vimentin, and Stat 3 was amplified using ABI Prism 7000 Sequence Detection System (Applied Biosystems). The primers for this study (Invitrogen) are shown in Table I. For all real-time PCR studies, negative controls were a non-reverse transcriptase reaction and a non-sample reaction (data not shown). Glyceraldehyde-3-phosphate dehydrogenase (GAPDH) was amplified as an internal standard. 
Table I. Primer sequences for amplification of human Interleukin-6, Vimentin, E-Cadherin, Stat3 or GAPDH.

\begin{tabular}{|c|c|c|c|c|}
\hline \multirow{2}{*}{$\begin{array}{l}\text { Gene } \\
\text { (human) }\end{array}$} & \multirow{2}{*}{$\begin{array}{c}\text { GeneBank } \\
\text { accession no. }\end{array}$} & \multicolumn{2}{|r|}{ Primers } & \multirow{2}{*}{$\begin{array}{l}\text { Product } \\
\text { size } \\
\text { (bp) }\end{array}$} \\
\hline & & Sense & Anti-sense & \\
\hline Interleukin-6 & NM_000600 & 5'-TCTCGAGAGCCCAGCTATGAACTC-3' & 5'-ATAGCGGCCGCTTACTACATTTGCCGAAGA-3' & 648 \\
\hline Vimentin & NM_003380 & 5'-GACAATGCGTCTCTGGCACGTCTT-3' & 5'-TCCTCCGCCTCCTGCAGGTTCTT-3' & 229 \\
\hline E-Cadherin & NM_044560 & 5'-CCCATCAGCTGCCCAGAAAATGAA-3' & 5'-CTGTCACCTTCAGCCATCCTGTTT-3' & 174 \\
\hline Stat3 & NM_213662 & 5'-TTGCCAGTTGTGGTGATC-3' & 5'-AGAACCCAGAAGGAGAAGC-3' & 313 \\
\hline GAPDH & NM_002046 & 5'-TGCACCACCAACTGCTTAGC-3' & 5'-GGCATGGACTGTGGTCATGAG-3' & 66 \\
\hline
\end{tabular}

Western blotting. HeLa and C33A cell lysates $(1 \mathrm{mg} / \mathrm{ml})$ were run on $12 \%$ sodium dodecyl sulfate/polyacrylamide gels under non-reducing conditions and transferred to nitrocellulose. After blocking the membrane with $3 \%$ non-fat milk, the membrane was incubated with monoclonal or polyclonal anti-IL-6 (1:500), anti-IL-6 receptor (1:1000), p-Stat3 (1:1000), Stat3 (1:2000), E-Cadherin (1:500), Vimentin (1:1000), p-Slug (1: 500$)$ and $\beta$-actin $(1: 100)$ overnight at $4^{\circ} \mathrm{C}$. The membrane was then incubated for $1 \mathrm{~h}$ with HRP-linked anti-mouse or anti-rabbit IgG (1:5000) diluted in TBST (10 mmol/l Tris- $\mathrm{HCl}$, $\mathrm{pH} 8.0$, containing $150 \mathrm{mmol} / 1 \mathrm{NaCl}$ and $0.1 \%$ Tween-20) and washed extensively with TBST and detected using the enhanced chemiluminescence system. The membranes were exposed to X-ray film (Amersham Biosciences). Intensities of immunoreactive bands were quantified using image analysis software (Scion).

Transient transfection of small interfering RNA. HeLa and C33A cells were transfected with small interfering RNA (siRNA) of Stat 3 (Invitrogen) and scrambled oligonucleotide siRNA as a negative control using lipofectamine RNAiMAX (Invitrogen) according to the manufacturer's protocols. Transfection efficiency was confirmed by western blot analysis.

Statistical analysis. All data were assessed using the Student's t-test. Levels of statistical significance were set at $\mathrm{P}<0.05$.

\section{Results}

Immunohistochemical features of human CSCC tissues. To examine immunohistochemical characteristics of human CSCC, 10 normal human cervical epithelia and 16 human CSCC tissues were randomly selected for this study. Welldifferentiated and poorly-differentiated human CSCC were diagnosed by two pathologists. H\&E staining showed histological features of well-differentiated CSCC as compared to normal cervical epithelium (Fig. 1A, upper panel). Immunofluorescence staining for Ki67 in well-differentiated CSCC tissues showed that the majority of Ki67-positive cells (indicated by red nuclear staining) are restricted to the basal and parabasal layers (Fig. 1A, right middle panel). Immunofluorescence staining for CDK4 showed a uniform cytoplasmic and nuclear staining in well-differentiated CSCC tissues (Fig. 1A, right middle panel). Strong nuclear staining for Cyclin D1 was observed in well-differentiated CSCC tissues (Fig. 1A, right bottom panel). The normal cervical epithelia were either completely non-immunoreactive or exhibited weak immunoreactivity (Fig. 1A, left panels). Cytokeratin (CK) is one of intermediate filament proteins and has been used as epithelial-developed cancer marker (24). To examine expression patterns of cytokeratin in human CSCC, well-differentiated and poorly-differentiated CSCC tissues were selected for staining with anti-CK8 and anti-CK14 antibodies. The significant positive staining for CK8 and CK14 was observed in all well-differentiated (Fig. 1B, bottom panel) and a few poorly-differentiated (Fig. 1B, upper panel) CSCC tissues. Both CK8 and CK14 showed more extensive and intense expression in well-differentiated CSCC tissues (Fig. 1B, bottom panel) as compared to poorly-differentiated CSCC tissues (Fig. 1B, upper panel). The p63 is a member of p53 family. Studies have shown that p63 plays an important role in the development and differentiation of various epithelial cells (25). To determine the expression of p63 in human CSCC, well-differentiated CSCC tissues were selected for immunofluorescence staining with anti-p63 antibody. The p63 was expressed robustly in well-differentiated CSCC tissues (Fig. 1C, upper panel), and colocalization of p63 and $\mathrm{Ki67}$ in cancer cells was demonstrated to significantly induce proliferation potential (Fig. 1C, bottom panel). Quantitative analysis shows that $60 \%$ of p63 positive cancer cells were colocalized with Ki67 in well-differentiated CSCC tissues (Fig. 1D). These data indicate that cell cycle-associated proteins, cytokeratins and p63 as immunohistochemical markers are highly expressed in well-differentiated human CSCC tissues.

Expression of IL-6R, Stat3, E-Cadherin and Vimentin in human CSCC tissues. IL-6 has been reported to play an important role in tumor progression and metastasis in a variety of human cancers as a central proinflammatory factor. To examine the expression of IL-6R and its downstream protein Stat 3 in human CSCC tissues, IL-6R and Stat 3 expression were analyzed using immunofluorescence staining. Both IL-6R $\alpha$ and IL-6R $\beta$ staining were observed in cancer lesions in 15 out of $16(93.8 \%$ ) of human CSCC tissues (Fig. 2A, right upper panels), whereas the negative control showed lack of staining for IL-6R $\alpha$ and IL-6R $\beta$ in normal cervical tissues and the positive staining for IL-6R $\alpha$ and IL-6R $\beta$ was restricted to the most basal and parabasal cells of normal squamous epithelium (Fig. 2A, left upper panels). Similarly, staining of Stat 3 and p-Stat 3 was observed in cancer lesions in 14 out of $16(87.5 \%)$ of the human CSCC tissues (Fig. 2A, right bottom 


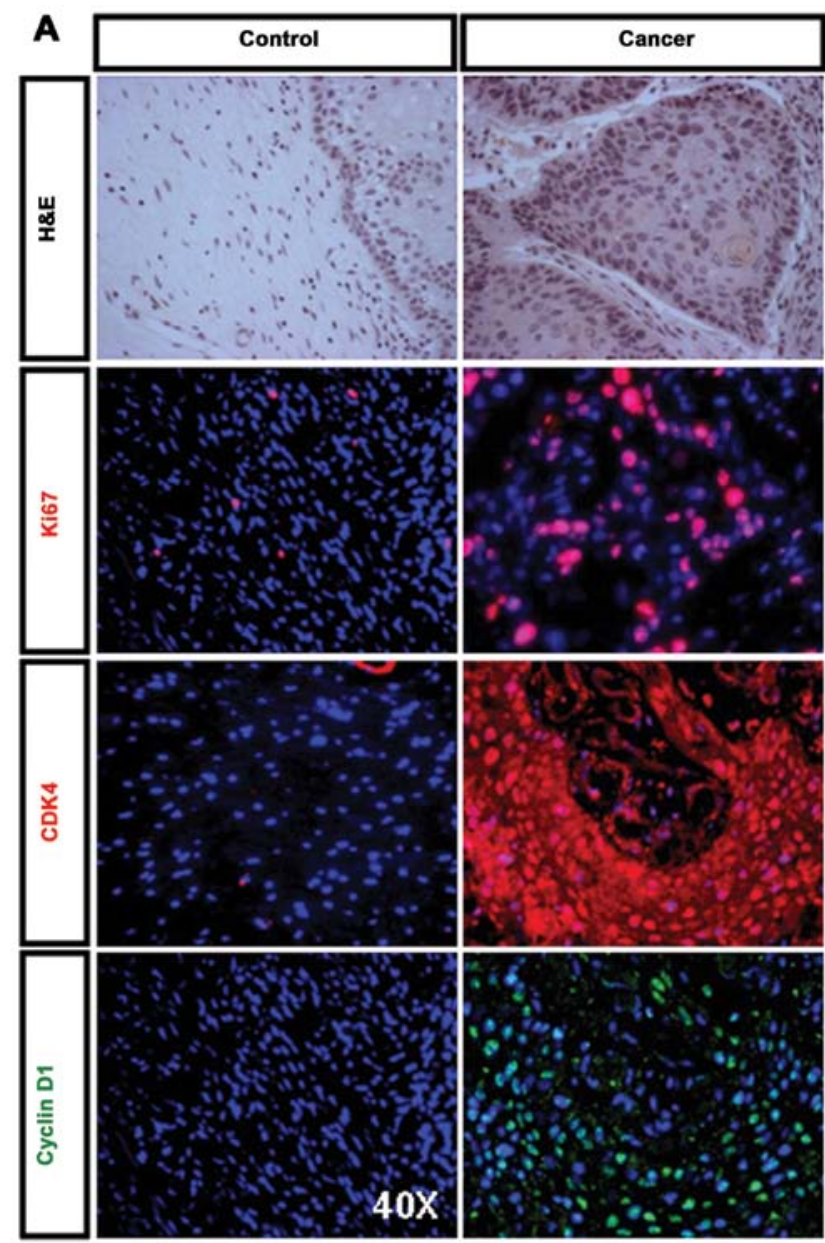

B

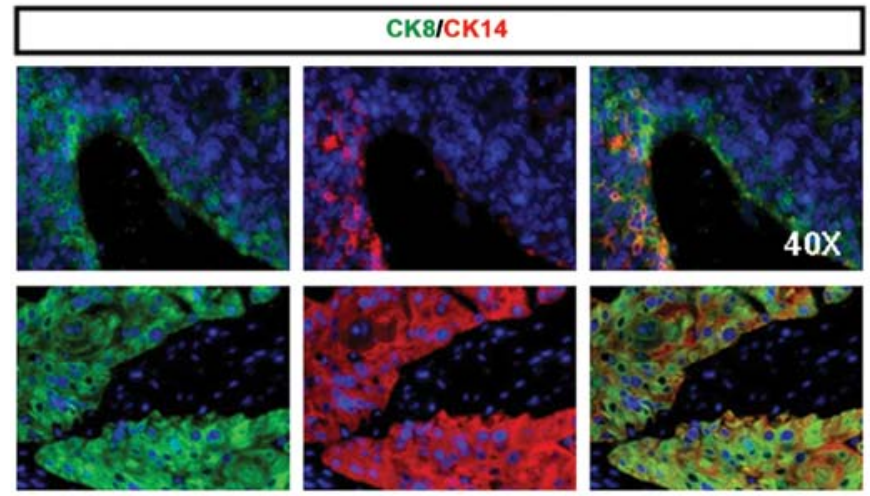

C
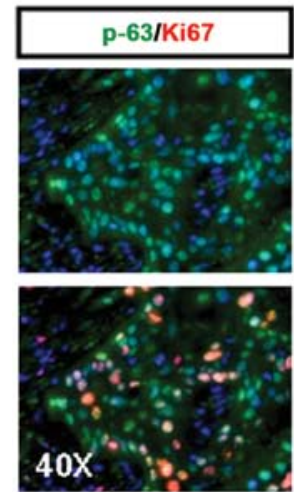

D

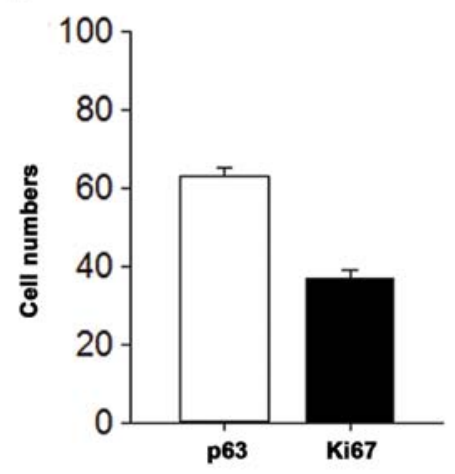

Figure 1. Immunohistochemical staining of Ki67, CDK4, Cyclin D1, CK8, CK14, p63 proteins in human CSCC tissues. (A) H\&E staining showed histological features of normal (left upper panel) and well-differentiated CSCC (right upper panel) tissues. The majority of Ki67 positive cells in CSCC tissues were restricted to the basal and parabasal layers (right middle panel). A uniform cytoplasmic and nuclear staining of CDK4 was detected in CSCC tissues (right middle panel). Strong nuclear staining for Cyclin D1 was observed in CSCC tissues (right bottom panel). The normal cervical epithelia were either completely non-immunoreactive or exhibited weak immunoreactivity. (B) CK8 and CK14 positive staining was observed in all well-differentiated (bottom panel) and a few poor-differentiated (upper panel) CSCC tissues. Both CK8 and CK14 showed more extensive and intense expression in well-differentiated CSCC tissues (bottom panel). (C) The p63 was expressed robustly in well-differentiated CSCC tissues (upper panel), and was colocalized with Ki67 in proliferated cancer cells (bottom panel). (D) Quantitative analysis shows that $60 \%$ of p63 expression was colocalized with Ki67 in well-differentiated CSCC tissues. Nuclei were stained with 4',6'-diamidino-2-phenylindole (DAPI) (blue) (original magnification, x400).

panels), whereas the negative control showed lack of staining for Stat 3 and p-Stat3 in normal cervical tissues (Fig. 2A, left bottom panels). EMT is characterized by dramatic phenotypic changes in human cancers. To evaluate expression patterns of EMT in human CSCC tissues, both well-differentiated and poorly-differentiated CSCC tissues were selected for immunofluorescence staining with epithelial marker E-Cadherin and mesenchymal marker Vimentin. E-Cadherin was absent in poorly-differentiated CSCC tissues (Fig. 2B, bottom panel), but was markedly observed in well-differentiated CSCC tissues (Fig. 2B, upper panel). In contrast, Vimentin was absent in well-differentiated CSCC tissues (Fig.2-B, upper panel), but was clearly expressed in poorly-differentiated CSCC tissues (Fig. 2B, bottom panel). These data indicate that IL-6R and p-Stat 3 are positively expressed in human CSCC tissues, and well-differentiated CSCC expresses high levels of E-Cadherin and low levels of Vimentin, whereas poorly-differentiated CSCC expresses high levels of Vimentin and low levels of E-Cadherin.
IL-6 stimulation induces IL-6 receptor expression in HeLa and C33A cells. To examine expression level of IL-6 receptor in HeLa and C33A cells. We first investigated protein expression levels of endogenous IL-6, IL-6 receptor, p-Stat3, E-Cadherin, Vimentin and p-Slug using western blot analysis. The expression of IL-6, IL-6R and p-Stat 3 proteins was significantly weaker in C33A cells as compared to HeLa cells (Fig. 3A). Similarly, a low level in endogenous p-Slug protein was observed in C33A cells as compared to HeLa cells (Fig. 3A). However, protein levels of endogenous E-Cadherin and Vimentin were not significantly different in both HeLa and C33A cells (Fig. 3A). We next examined expression levels of IL-6R protein and mRNA in HeLa and C33A cells in response to IL-6 as compared to control and IL-6 + curcumin for $48 \mathrm{~h}$ using western blot analysis and real-time RT-PCR. The expression levels of IL-6R protein and mRNA were significantly increased in IL-6-treated HeLa (Fig. 3B and C, quantitative results) and C33A (Fig. 3D and E, quantitative results) cells as comparing to control and IL-6 + curcumin. 


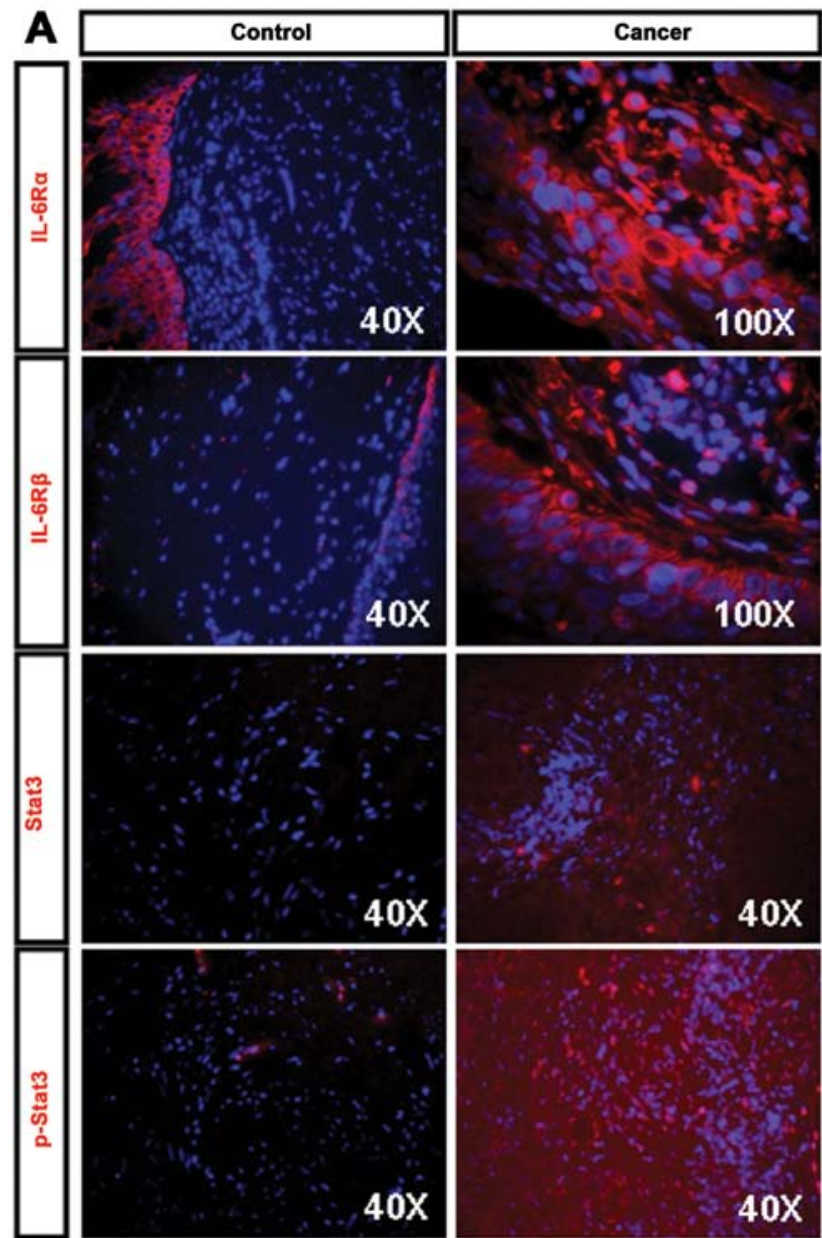

These data indicate that expression level of IL-6R is similar in response to IL-6 stimulation in HeLa and C33A cells, while endogenous IL-6R and p-Stat 3 are weakly expressed in C33A cells.

Treatment of IL-6 mediates cell proliferation and alters cell morphology. There is increasing evidence to show that IL-6 is a regulator of cancer cell proliferation (26). To examine whether IL-6 mediates growth of human cervical carcinoma cells, HeLa and C33A cells were treated with IL-6 or IL-6 + curcumin for 48 h. Cells were stained with anti-Ki67 antibody (Fig. 4A). The cell proliferation was significantly mediated by IL- 6 stimulation in IL-6-treated HeLa and C33A cells as compared to control and IL-6 + curcumin treatment groups (Fig. 4A and B, quantitative results). To examine whether IL-6 mediates changes in cell morphology, we treated HeLa cells with IL- 6 for 48 h. In the absence of IL- 6 treatment, HeLa cells exhibited well-organized cell-cell association and islet-like structure, and characteristics of squamous cell carcinoma (Fig. 4C, upper panel). Interestingly, IL-6 treatment induced cell elongation and increased scattering, resulting in a similar morphology to mesenchymal cells (Fig. 4C, bottom panel). These data indicate that IL- 6 is able to mediate cell proliferation and alter cell morphology in HeLa cells.

IL-6 stimulation induces the EMT program in HeLa and C33A cells. EMT is a process by which epithelial cells lose their cell
B

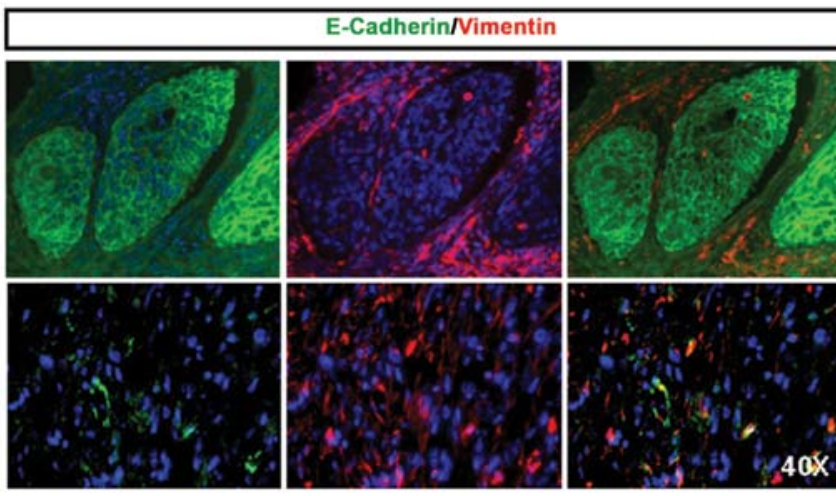

Figure 2. Expression of IL-6R, Stat3, E-Cadherin and Vimentin in human CSCC tissues. (A) Human normal cervical and well-differentiated CSCC tissues were stained using anti-IL-6R $\alpha$, IL-6R $\beta$ Stat 3 and p-Stat 3 antibodies. Strong staining of IL-6R $\alpha$ and IL-6R $\beta$ was detected on the cancer cell membrane in CSCC tissues (right upper and middle panels), whereas the positive staining of IL-6R $\alpha$ and IL-6R $\beta$ was only restricted in squamous epithelium in normal cervical tissues (left upper and middle panels). The expression of Stat 3 was weakly detected in CSCC tissues (right middle panel), whereas p-Stat 3 showed strong nuclear expression in CSCC tissues (right bottom panel) as comparing to normal cervical tissues (left middle and bottom panels). Nuclei were stained with DAPI (blue) (original magnification, $x 400$ and $\mathrm{x} 1000$ ). (B) E-Cadherin was weakly expressed in poorly-differentiated human CSCC tissues (bottom panel), but it was markedly observed in welldifferentiated human CSCC tissues (upper panel). In contrast, Vimentin was lost in well-differentiated human CSCC tissues (upper panel), but it was clearly expressed in poorly-differentiated human CSCC tissues (bottom panel). Nuclei were stained with DAPI (original magnification, $x 400$ ).

polarity and cell-cell adhesion, and gain migratory and invasive properties to become mesenchymal cells. Many factors have been reported to induce EMT program in cancer progression and metastasis (27). To evaluate whether EMT program caused by IL- 6 exposure in human cervical carcinoma, we first examined the expression of EMT markers in HeLa cells by immunofluorescence staining. Cells treated with IL-6 for $48 \mathrm{~h}$ decreased E-Cadherin expression, but significantly increased Vimentin expression as comparing to the cells treated with IL-6 + curcumin (Fig. 5A). Similar effects of E-Cadherin and Vimentin were observed in C33A cell line(Fig.5D).Interestingly, a transition to spindle-shaped morphology was also observed in HeLa cells (Fig. 5A), but not in C33A cells (Fig. 5D). We next determined the expression levels of E-Cadherin and Vimentin in HeLa cells in response to IL- 6 treatment for $48 \mathrm{~h}$ by western blot analysis and real-time RT-PCR. The protein and mRNA levels of E-Cadherin were significantly decreased in HeLa cells treated with IL-6 as comparing to HeLa cells treated with DMSO and IL-6 + curcumin (Fig. 5B). In contrast, the protein and mRNA levels of Vimentin markedly increased in HeLa cells in response to IL- 6 treatment as comparing to HeLa cells treated with DMSO and IL-6 + curcumin (Fig. 5C). Similar effects of E-Cadherin and Vimentin were observed in C33A cells (Fig. 5E and F). These results indicate that IL-6 exhibits an important role on the EMT induction in human cervical carcinoma cells by downregulation of epithelial markers and upregulation of mesenchymal markers.

Treatment of HeLa and C33A cells with IL-6 increases phosphorylation of Stat3. Stat3 is a downstream protein of IL-6R 
and plays a potential role in cervical cancer development. To investigate whether IL-6 directly activates Stat3 in human cervical carcinoma, we examined the expression of $\mathrm{p}$-Stat 3 in

A
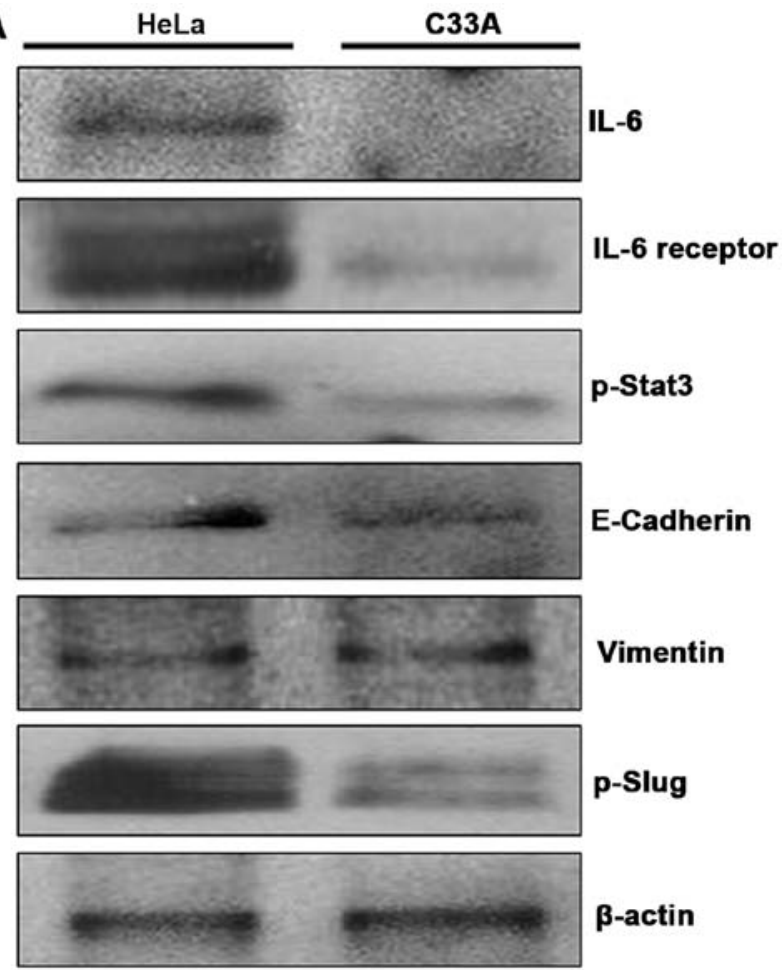

HeLa

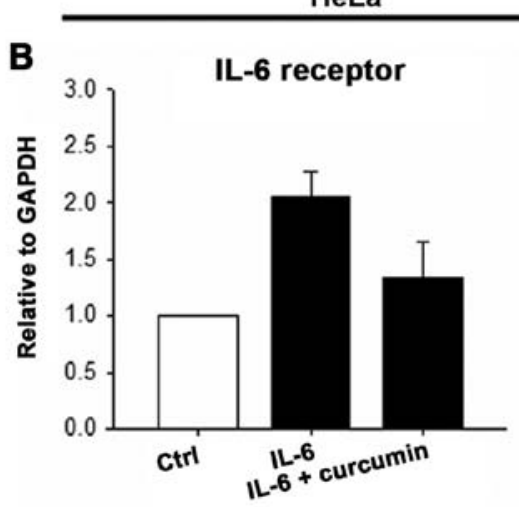

C

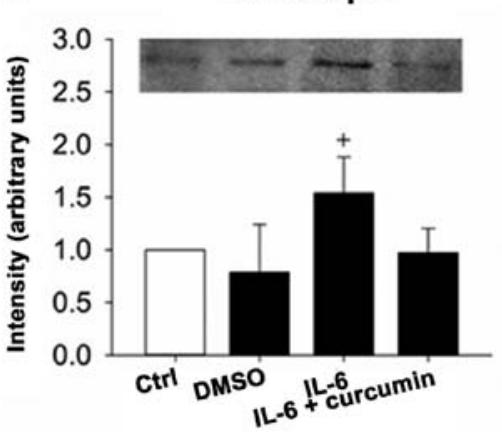

HeLa cells after treatment with IL- 6 for $48 \mathrm{~h}$. IL-6 treatment significantly increased the expression of p-Stat 3 as compared to control and IL- 6 + curcumin groups as demonstrated by immunofluorescence (Fig. 6A, quantitative results). Also, an increase in p-Stat 3 protein levels was observed in HeLa cells treated with various concentrations of IL- 6 for $48 \mathrm{~h}$ and was significant difference at concentrations of 50 and $200 \mathrm{ng} / \mathrm{ml}$ (Fig. 6B, quantitative results). Similar effect of p-Stat 3 expression by IL-6 stimulation was observed by real-time RT-PCR in HeLa cells (data not shown). Stat 3 and p-Stat 3 protein levels were then measured by western blot analysis following treatment of HeLa cells with IL-6 and IL-6 + curcumin for $48 \mathrm{~h}$. No changes in total Stat 3 levels were noted at $48 \mathrm{~h}$ in treated cells as comparing to control, DMSO and IL- 6 + curcumin groups (Fig. 6C). In contrast, IL-6 stimulation significantly increased expression of $\mathrm{p}$-Stat 3 protein as comparing to control, DMSO and IL-6 + curcumin groups (Fig. 6C, quantitative results).

We next determined the p-Stat 3 expression in C33A cells in response to IL- 6 treatment for $48 \mathrm{~h}$ by immunofluorescence and western blot analysis. IL- 6 treatment significantly increased the expression of p-Stat 3 in C33A (Fig. 6D). In addition, the $\mathrm{p}$-Stat 3 expression was increased in C33A cells treated with various concentrations of IL-6 for 48 h (Fig. 6E, quantitative results). The protein levels of p-Stat 3 were significantly increased in C33A cells treated with IL-6 as comparing to $\mathrm{C} 33 \mathrm{~A}$ cells treated with DMSO and IL-6 + curcumin (Fig. 6F, quantitative results). These results indicate that IL-6
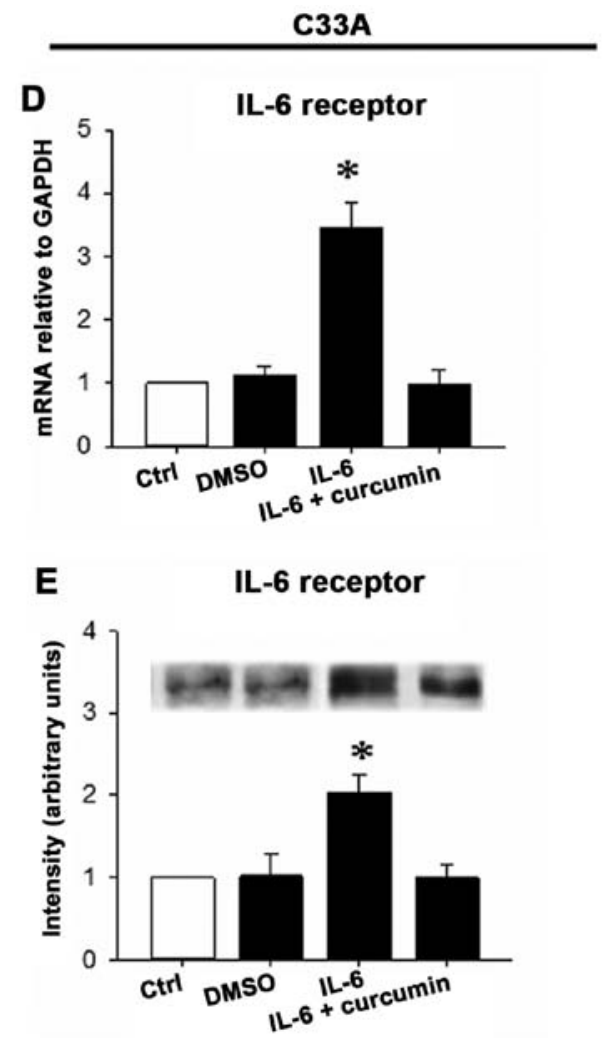

Figure 3. Expression of IL-6, IL-6R, p-Stat3, E-Cadherin, Vimentin and p-Slug in HeLa and C33A cells. (A) The protein expression levels of endogenous IL-6, IL-6R, p-Stat3 and p-Slug were weakly detected in C33A cells as compared to HeLa cells, but the protein levels of endogenous E-Cadherin and Vimentin were no different in HeLa and C33A cells. (B-E) The expression levels of IL-6R protein and mRNA were significantly increased in IL-6-treated HeLa and C33A cells for $48 \mathrm{~h}$ as compared to control, DMSO and IL- $6+$ curcumin groups (quantitative results). $\beta$-actin was used as an internal control. The intensity of bands was quantified using ImageJ software and normalized to $\beta$-actin. 


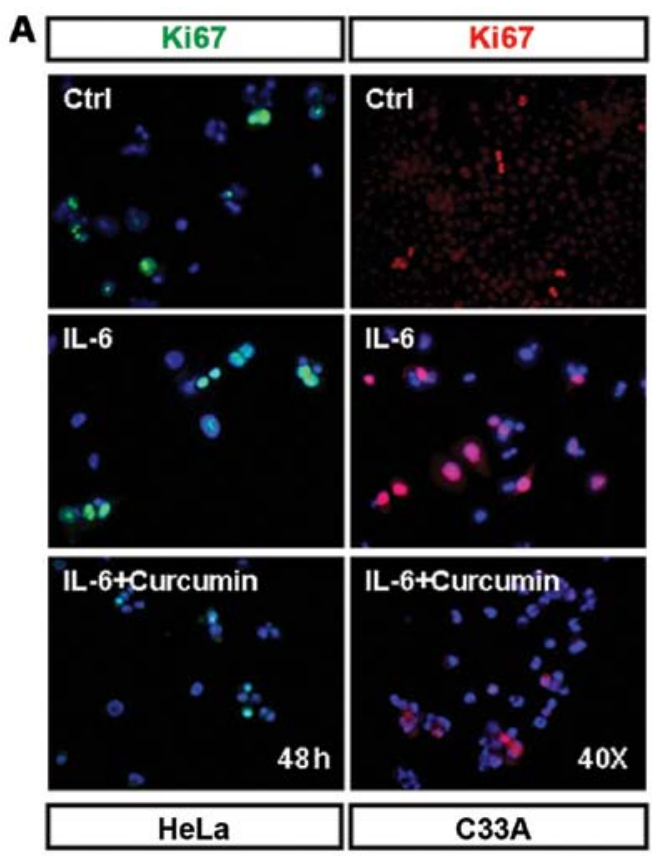

C

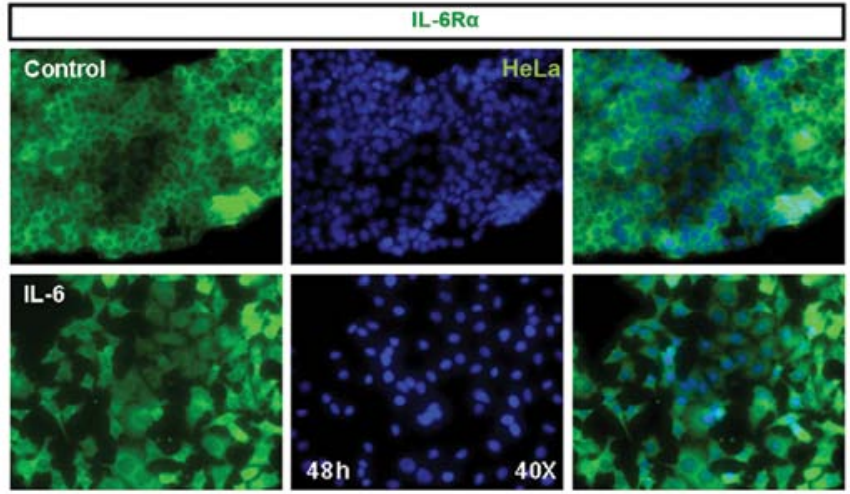

Figure 4. IL-6-induced cell proliferation and cell morphology alteration. (A) HeLa and C33A cells were treated without IL-6 (as a control) and with IL-6 $(50 \mathrm{ng} / \mathrm{ml})$, IL-6 + curcumin $(10 \mu \mathrm{M})$ for $48 \mathrm{~h}$. Cell proliferation was evaluated by immunostaining using Ki67 antibody. (B) Quantitative analysis shows that IL-6-induced HeLa and C33A cell proliferation as compared to control and IL-6 + curcumin group. (C) HeLa cells were treated with IL-6 for $48 \mathrm{~h}$, alteration of cell morphology was observed by immunostaining using IL-6R $\alpha$ antibody (bottom panel) as compared to control (upper panel). Nuclei were stained with DAPI (original magnification, $\mathrm{x} 400$ ).

can directly induce phosphorylation of Stat 3 in human cervical carcinoma cells, HeLa and C33A.

IL-6-induces the EMT program via Stat3 in HeLa and C33A cells. To evaluate the role of Stat3 in IL-6-induced EMT program, we examined expression of p-Stat3, E-Cadherin, Vimentin and p-Slug in HeLa and C33A cells treated with and without IL-6 for $48 \mathrm{~h}$ after knockdown of Stat3. The expression of p-Stat 3 was markedly decreased in HeLa cells treated with and without IL-6 as compared to control by western blot analysis (Fig. 7A). Similar effects were observed in C33A cell line (Fig. 7B). The Vimentin and E-Cadherin levels were then measured by western blot analysis following treatment of HeLa cells with and without IL-6 for $48 \mathrm{~h}$. The protein levels of E-Cadherin were significantly increased in HeLa

\section{B}

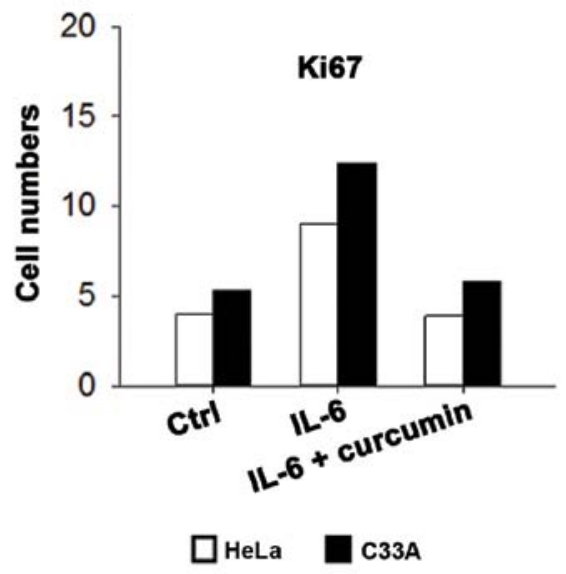

cells treated with IL-6 as compared to HeLa cells treated with control, but no difference for E-Cadherin was observed in HeLa cells untreated with IL-6 in control and siRNA groups (Fig. 7A). In contrast, the protein levels of Vimentin markedly decreased in HeLa cells in response to IL-6 treatment as compared to HeLa cells treated with control, but Vimentin levels were significantly decreased in HeLa cells untreated with IL-6 in siRNA group as comparing to control (Fig. 7A). Similar effects were observed in C33A cell line (Fig. 7B). IL-6 treatment in HeLa cells significantly decreased expression of $\mathrm{p}$-Slug protein in siRNA group as compared to control. In contrast, p-Slug levels were also decreased in HeLa cells untreated with IL-6 in siRNA group as compared to control (Fig. 7A). No changes in p-Slug protein levels were noted in C33A cells treated and untreated with IL-6 in control and siRNA groups (Fig. 7B). These results indicate that IL-6-induced E-Cadherin downregulation and Vimentin upregulation were dramatically blocked by knockdown of Stat3, suggesting that Stat3 may participate in IL-6-induced EMT program in HeLa and C33A cells.

\section{Discussion}

EMT is an important process in many types of human epithelial carcinoma including cervical carcinoma (19). Despite HPV infection has been reported to be a major risk for developing cervical carcinoma, however cervical carcinoma is also closely associated with inflammatory conditions $(3,4)$. Studies have shown that many Inflammation mediators can trigger EMT program in progression of human cancers. IL-6 is one of pro-inflammatory cytokines that activates its downstream protein Stat3 within the tumor microenvironment and plays an important role in the pathogenesis of human cervical carcinoma $(28,29)$. In this study, we investigated the role of IL-6 as an inducer of EMT through its downstream signaling molecule Stat 3 in human cervical carcinoma. 

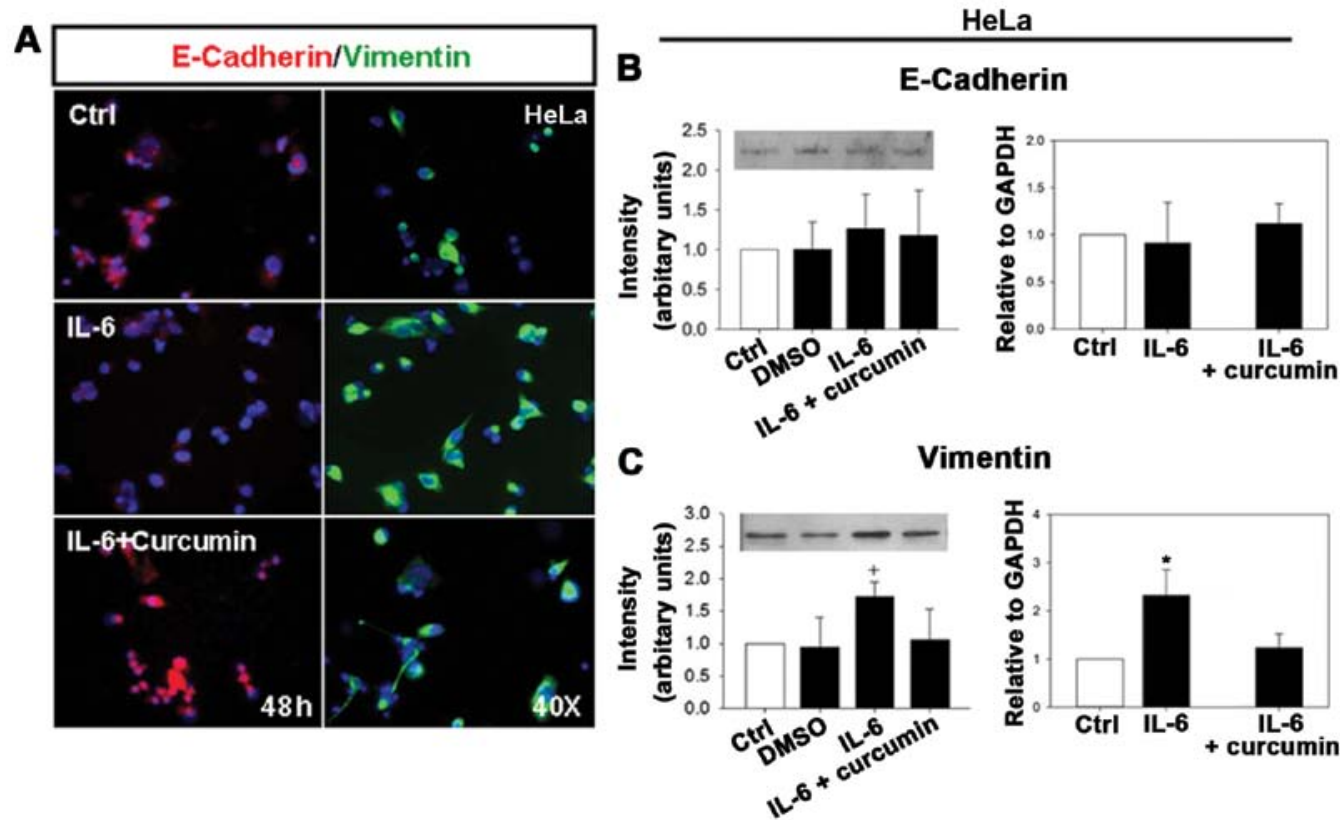

Vimentin

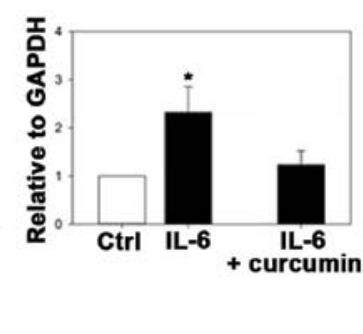

\section{C33A}
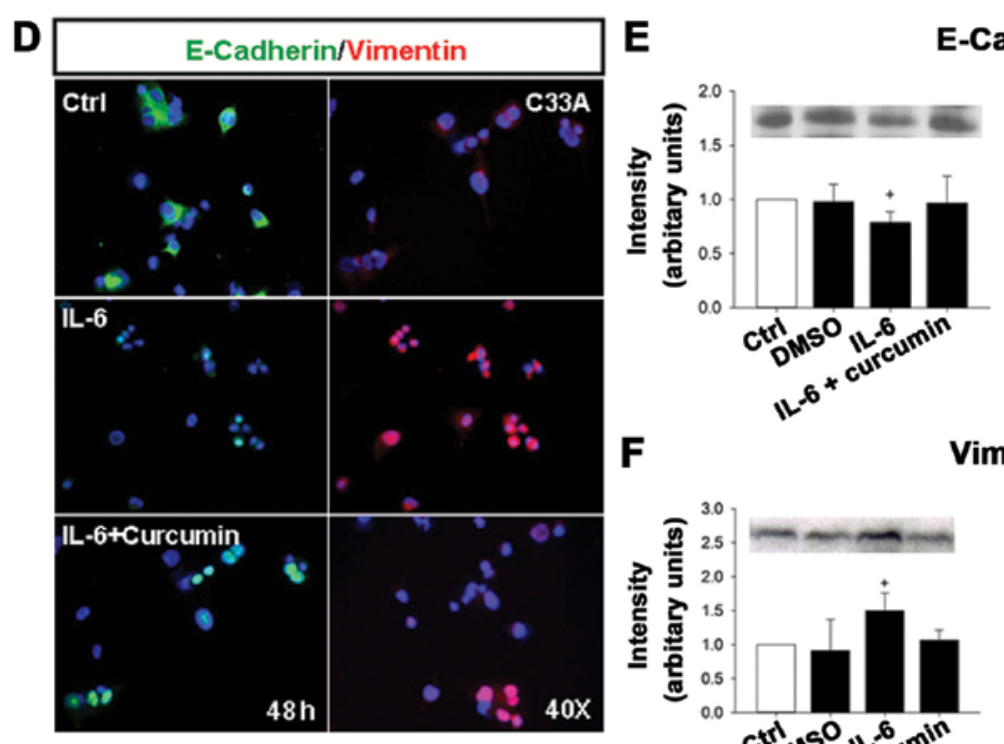

E-Cadherin
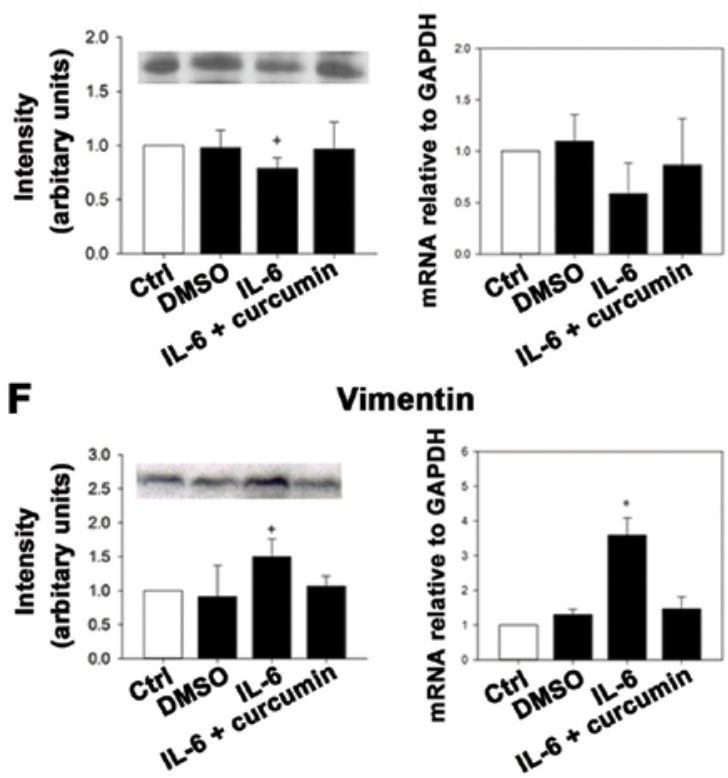

Vimentin

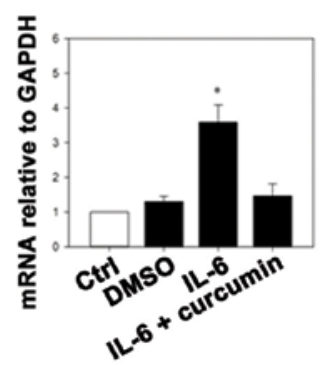

Figure 5. IL-6 treatment induces EMT in HeLa and C33A cells. (A) HeLa cells were treated without IL-6 (as a control) and with IL-6 (50 ng/ml), IL-6 + curcumin $(10 \mu \mathrm{M})$ for $48 \mathrm{~h}$. Cells were stained with anti-E-Cadherin antibody and rhodamine-conjugated secondary antibody (red) or stained with anti-Vimentin antibody and fluorescein isothiocyanate-conjugated secondary antibody (green). Nuclei were stained with DAPI (blue) (original magnification, $\mathrm{x} 400$ ). (B and C) HeLa cells were treated without IL-6 (as a control) and with DMSO, IL-6 $(50 \mathrm{ng} / \mathrm{ml})$ and IL-6 + curcumin $(10 \mu \mathrm{M})$ for 48 h. Total protein was extracted from control, DMSO and treated cells. The protein levels of E-Cadherin (B, left) and Vimentin (C, left) were analyzed using western blot analysis. Similarly, total RNA was extracted from control and treated cells. The mRNA levels of E-Cadherin (B, right) and Vimentin (C, right) were determined using real-time RT-PCR. Real-time PCR for GADPH served as an internal control. (D) C33A cells were treated without IL-6 (as a control) and with IL-6 (50 ng/ml), IL-6 + curcumin (10 $\mu \mathrm{M}$ ) for $48 \mathrm{~h}$. Cells were stained with anti-E-Cadherin antibody and rhodamine-conjugated secondary antibody (red) or stained with anti-Vimentin antibody and fluorescein isothiocyanate-conjugated secondary antibody (green). Nuclei were stained with DAPI (blue) (original magnification, $\mathrm{x} 400$ ). (E and F) C33A cells were treated without IL-6 (as a control) and with DMSO, IL-6 (50 ng/ml) and IL-6 + curcumin (10 $\mu \mathrm{M})$ for $48 \mathrm{~h}$. Total protein was extracted from control, DMSO and treated C33A cells. The protein levels of E-Cadherin (E, left) and Vimentin (F, left) were analyzed using western blot analysis. Similarly, total RNA was extracted from control and treated cells. The mRNA levels of E-Cadherin (E, right) and Vimentin (F, right) were determined using real-time RT-PCR. Real-time PCR for GADPH served as an internal control. The intensity of bands was quantified using ImageJ software and normalized to $\beta$-actin.

In human CSCC tissues, we observed overexpression of IL-6R signaling molecules and EMT markers, suggesting that these molecules and markers are necessary for development of human CSCC. Interestingly, the expression of epithelial cell marker E-Cadherin was more dominant than mesenchymal cell marker Vimentin in well-differentiated CSCC 

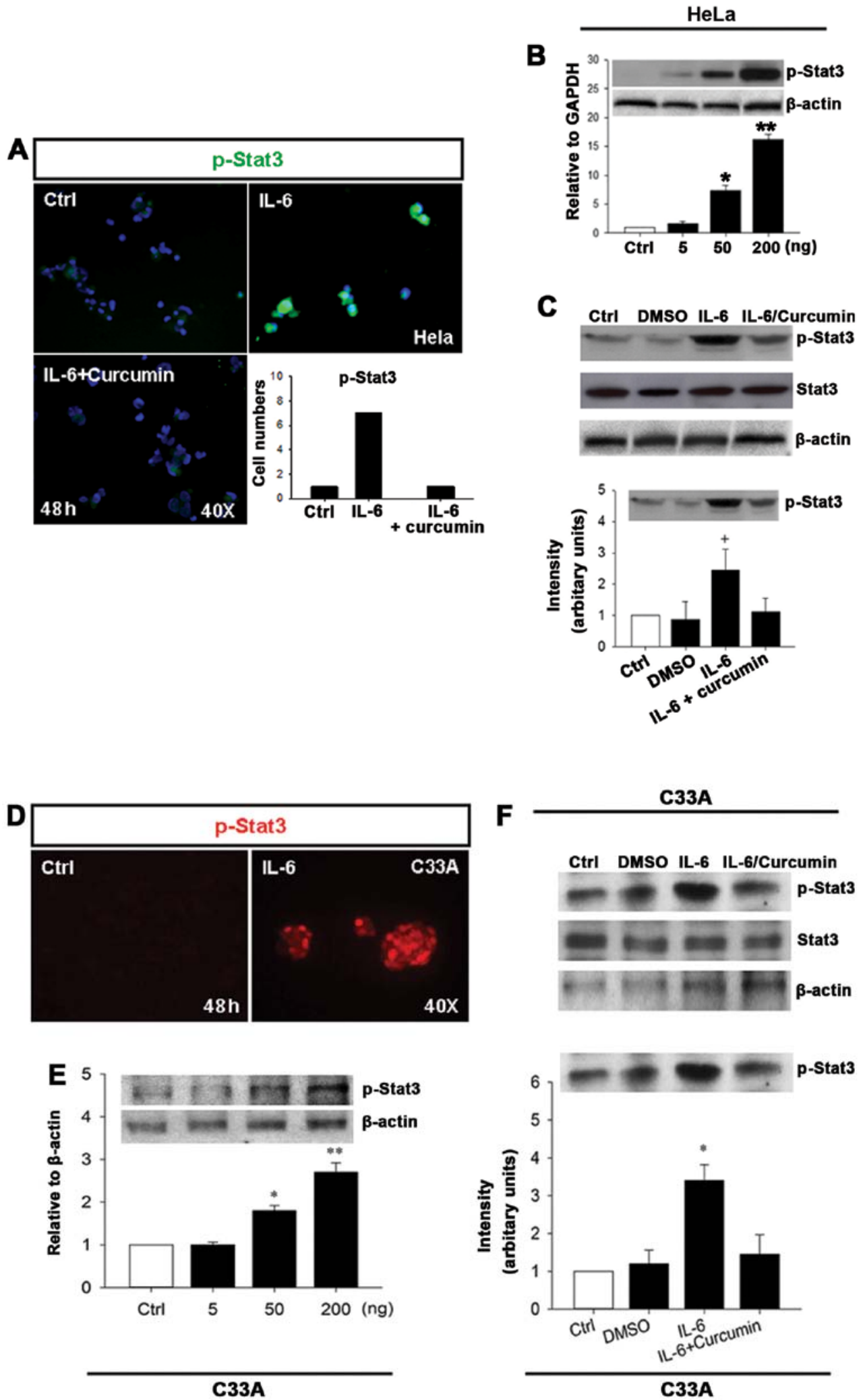

Figure 6. IL-6-induced phosphorylation of Stat3 by treatment with IL-6 in HeLa and C33A cells. (A) HeLa cells were treated with IL-6 (50 ng/ml) and IL-6 + curcumin $(10 \mu \mathrm{M})$ for $48 \mathrm{~h}$. HeLa cells were stained with anti-p-Stat3 (Y705) antibody and fluorescein isothiocyanate-conjugated secondary antibody (green). Nuclei were stained with DAPI (blue) (original magnification, x400). Quantitative analysis of p-Stat3 expression is markedly higher in IL-6 treated cells as compared to untreated and IL- $6+$ curcumin $(10 \mu \mathrm{M})$ treated cells. (B) HeLa cells were stimulated with IL-6 (5, 50 and $200 \mathrm{ng} / \mathrm{ml})$ and without IL-6 for $48 \mathrm{~h}$. Cell lysates were subjected to immunoblot analysis using anti-p-Stat 3 antibody and $\beta$-actin as loading control. (C) HeLa cells were treated without IL-6 and with DMSO, IL-6 $(50 \mathrm{ng} / \mathrm{ml})$ and IL-6 + curcumin $(10 \mu \mathrm{M})$ for $48 \mathrm{~h}$. Cell lysates were subjected to immunoblot analysis using anti-p-Stat3 and anti-Stat3 antibodies. $\beta$-actin was a loading control. (D) C33A cells were treated with IL-6 (50 ng/ml) for $48 \mathrm{~h}$. Cells were stained with anti-p-Stat3 (Y705) antibody and rhodamine-conjugated secondary antibody (red) (original magnification, $\mathrm{x} 400$ ). (E) C33A cells were stimulated with IL-6 (5, $50 \mathrm{and} 200 \mathrm{ng} / \mathrm{ml})$ and without IL-6 (as a control) for $48 \mathrm{~h}$. Cell lysates were subjected to immunoblot analysis using anti-p-Stat3 antibody and $\beta$-actin as a loading control. (F) C33A cells were treated without IL-6 (as a control) and with DMSO, IL-6 (50 ng/ml) and IL-6 + curcumin $(10 \mu \mathrm{M})$ for 48 h. Cell lysates were subjected to immunoblot analysis using anti-p-Stat 3 and anti-Stat 3 antibodies. $\beta$-actin was used as a loading control. The intensity of bands was quantified using ImageJ software and normalized to $\beta$-actin. 
A

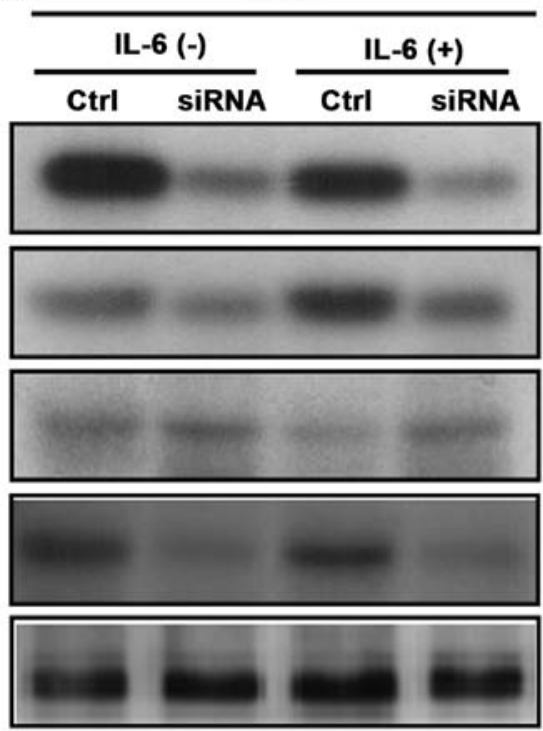

B

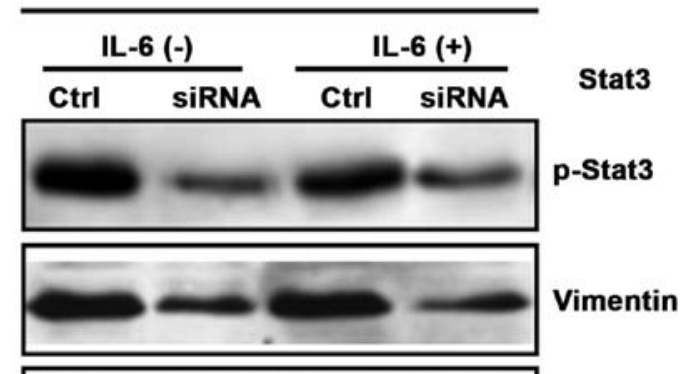

E-Cadherin

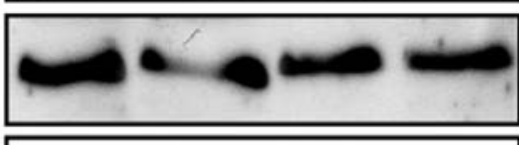

p-Slug

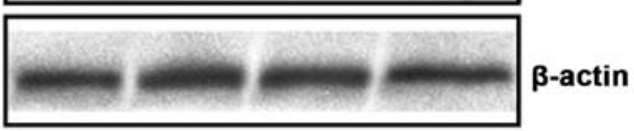

Figure 7. Stat3 knockdown significantly reverses the IL-6-induced EMT program in HeLa and C33A cells. (A) Stat 3 was knocked down in HeLa cells by respective siRNA. Cells treated with or without IL- 6 for $48 \mathrm{~h}$ and whole-cell extracts were analyzed by SDS-PAGE. The protein expression levels of p-Stat3, E-Cadherin, Vimentin and p-Slug were examined by western blot analysis. Equal protein loading was verified by stripping the blots and reprobing with $\beta$-actin antibody. (B) Stat 3 was knocked down in C33A cells by respective siRNA. Cells were treated with or without IL-6 for $48 \mathrm{~h}$, and cell lysates were subjected to immunoblot analysis using anti-p-Stat3, anti-E-Cadherin, anti-Vimentin and anti-p-Slug antibodies. The intensity of bands was quantified using ImageJ software and normalized to $\beta$-actin as a loading control.

tissues, whereas Vimentin was more strongly expressed in poorly-differentiated CSCC tissues than E-Cadherin. This is consistent with data obtained from Cheng et al (30). Further evidence from cytokeratins (CK8 and CK14) and p63 staining support that the expression of epithelial cell markers was the major phenotype in well-differentiated human CSCC tissues. Our data show that $60 \%$ of p63 positive cancer cells were colocalized with Ki67 in well-differentiated CSCC tissues, suggesting that the colocalization of p63 and Ki67 in cancer cells was demonstrated to significantly induce proliferation potential. We also observed that the expression of N-Cadherin and $\beta$-Catenin (data not shown) was significantly increased in well-differentiated human CSCC tissues. E-Cadherin and N-Cadherin are known to anchor and sequester $\beta$-Catenin on the cell membrane and preventing its activation; the activation of $\beta$-Catenin may result from the upregulation of E-Cadherin and the downregulation of $\mathrm{N}$-Cadherin (31). Moreover, we observed a loss of Vimentin in well-differentiated CSCC lesion and a strong expression of Vimentin in the connective tissues surrounding the CSCC lesion. However, we do not know whether EMT can be triggered directly by IL-6 in human CSCC tissue, because we were unable to examine the relationship between IL- 6 and EMT program using human tumor tissues.

To identify whether IL-6 is involved in EMT induction and development of cervical carcinoma, HeLa, an HPV-positive cervical cancer cell line and C33A, an HPV-negative cervical cancer cell line were chosen for in vitro experiment. Interestingly, both HeLa and C33A cells treatment with IL-6 promoted IL-6R expression, cell proliferation, and morphology alteration, despite the expression levels of endogenous IL-6, IL-6R and p-Stat 3 were weaker in C33A cells as comparing to
HeLa cells. One possibility we consider is that the activation of IL-6R signaling may not be dependent on HPV infection. We found that IL-6 treatment in HeLa and C33A cells decreased the expression of epithelial cell markers such as E-Cadherin and $\beta$-Catenin (data not shown), but increased the expression of mesenchymal cell markers such as Vimentin and N-Cadherin (data not shown). Interestingly, a transition to spindle-shaped morphology was detectable in HeLa cells, but not in C33A cells. We also found that Stat 3 was upregulated upon IL-6 stimulation in both HeLa and C33A cells. Furthermore, induction of Stat 3 by IL- 6 was successfully inhibited by the IL-6R inhibitor curcumin, indicating that IL-6-mediated Stat3 induction and subsequent EMT are dependent on IL-6R activation. Although Sullivan et al (32) have reported that IL-6 can induce EMT change in human breast cancer cells through Stat 3 activation, whether Stat 3 plays a role in EMT induction in human cervical carcinoma still remains to be determined. In our present study, we found that knockdown of Stat3 reversed IL-6-induced EMT program in cervical carcinoma cells, suggesting that Stat3 may be involved in EMT change and may be one of the mechanisms responsible for the regulation of IL-6R expression. Slug has been reported as a major transcriptional repressor to downregulate E-Cadherin expression resulted in initiating events in EMT (33). In our study, we further found that p-Slug expression was significantly decreased by knockdown of Stat3 in HeLa cells, but not in C33A cells, suggesting that the activation of Stat 3 is important for Slug expression in human cervical carcinoma. However, we do not know why p-Slug expression in HeLa cells and C33A cells is different after knockdown of Stat3. Therefore, our data indicated that IL- 6 is a novel inducer of EMT via Stat 3 in human cervical carcinoma cell lines, HeLa and $\mathrm{C} 33 \mathrm{~A}$. 
Our study provides new insight into the regulation of EMT by IL-6 via activation of Stat 3 in human cervical carcinoma. First, our data demonstrate that epithelial cell markers, CK8, CK14 and p63, showed more extensive and intense expression in well-differentiated CSCC lesion with epithelial morphology, suggesting that these markers, like E-Cadherin, may play important roles in the development of human cervical carcinoma. Second, our data indicate that knockdown of Stat 3 resulted in upregulation of E-Cadherin and downregulation of Vimentin in cervical carcinoma cells, suggesting that IL-6 may participate in Stat3-induced Slug expression in HeLa cells, and may mediate Stat3-induced downregulation of E-Cadherin.

The present study also has some limitations. Welldifferentiated and poorly-differentiated human CSCC tissues were surgically collected from patients rather than radiotherapy or chemotherapy. However, the problem with this is that by only studying tissues from a few well-differentiated and poorly-differentiated human CSCC, determining the role that IL- 6 may be playing throughout disease progression is limited. Therefore, further studies using larger patient populations are necessary to explore the role of IL-6 for different types of cervical carcinoma in order to obtain more reliable results.

In conclusion, our results reveal that IL-6 induced the EMT program in human cervical carcinoma cells via the activation of Stat3, and Stat3 knockdown significantly reversed IL-6-induced EMT program. These results provide a mechanistic explanation for Stat 3 as an important player in IL-6-driven EMT program, it is possible that blockage of Stat 3 may be a good approach to treat human cervical carcinoma that are driven by IL-6/IL-6R signaling. Overall, our findings indicate that Stat3 plays an important role in IL-6induced EMT and may serve as an attractive therapeutic target for human cervical carcinoma driven by IL-6/IL-6R signaling.

\section{Acknowledgements}

This study was supported by a grant from The Scientific Research Foundation for Returned Overseas Chinese Scholars, Bureau of Human Resources and Social Security of Beijing, P.R. China to J.-W.M. (grant no. 2008002).

\section{References}

1. Thun MJ, Oliver DeLancey J, Center MM, Jemal A and Ward EM: The global burden of cancer: priorities for prevention. Carcinogenesis 31: 100-110, 2010.

2. Lee MY and Shen MR: Epithelial-mesenchymal transition in cervical carcinoma. Am J Transl Res 4: 1-13, 2012.

3. Castellsague X: Natural history and epidemiology of HPV infection and cervical cancer. Gynecol Oncol 110: S4-S7, 2008.

4. Burd EM: Human papillomavirus and cervical cancer. Clin Microbiol Rev 16: 1-17, 2003.

5. zur Hausen H: Papillomaviruses and cancer: from basic studies to clinical application. Nat Rev Cancer 2: 342-350, 2002.

6. Au WW, Abdou-Salama S, Sierra-Torres $\mathrm{CH}$ and Al-Hendy A: Environmental risk factors for prevention and molecular intervention of cervical cancer. Int J Hyg Environ Health 210: 671-678, 2007.

7. Wei LH, Kuo ML, Chen CA, Cheng WF, Cheng SP, Hsieh FJ and Hsieh CY: Interleukin-6 in cervical cancer: the relationship with vascular endothelial growth factor. Gynecol Oncol 82: 49-56, 2001.
8. Wei LH, Kuo ML, Chen CA, Chou CH, Cheng WF, Chang MC, Su JL and Hsieh CY: The anti-apoptotic role of interleukin-6 in human cervical cancer is mediated by up-regulation of Mcl-1 through a PI3-K/Akt pathway. Oncogene 20: 5799-5809, 2001.

9. Rose-John S: IL-6 trans-signaling via the soluble IL-6 receptor: importance for the pro-inflammatory activities of IL-6. Int J Biol Sci 8: 1237-1247, 2012.

10. Braunstein J, Brutsaert S, Olson R and Schindler C: Stat dimerize in the absence of phosphorylation. J Biol Chem 278: 34133-34140, 2003.

11. Shukla S, Shishodia G, Mahata S, et al: Aberrant expression and constitutive activation of Stat 3 in cervical carcinogenesis: implications in high-risk human papillomavirus infection. Mol Cancer 9: 282, 2010.

12. Chen CL, Hsieh FC, Lieblein JC, et al: Stat 3 activation in human endometrial and cervical cancers. Br J Cancer 96: 591-599, 2007.

13. Aggarwal BB, Kunnumakkara AB, Harikumar KB, et al: Signal transducer and activator of transcription-3, inflammation, and cancer: how intimate is the relationship? Ann N Y Acad Sci 1171: 59-76, 2009.

14. Lee JM, Dedhar S, Kalluri R and Thompson EW: The epithelialmesenchymal transition: new insights in signaling, development, and disease. J Cell Biol 172: 973-981, 2006.

15. Trimboli AJ, Fukino K, Bruin A, et al: Direct evidence for epithelial-mesenchymal transitions in breast cancer. Cancer Res 68: 937-945, 2008

16. Vergara D, Merlot B, Lucot JP, Collinet P, Vinatier D, Fournier I and Salzet M: Epithelial-mesenchymal transition in ovarian cancer. Cancer Lett 291: 59-66, 2009.

17. Brabletz T, Hlubek F, Spaderna S, Schmalhofer O, Hiendlmeyer E, Jung $\mathrm{A}$ and Kirchner T: Invasion and metastasis in colorectal cancer: epithelial-mesenchymal transition, mesenchymal-epithelial transition, stem cells and beta-catenin. Cells Tissues Organs 179: 56-65, 2005.

18. Usami Y, Satake S, Nakayama F, et al: Snail-associated epithelial-mesenchymal transition promotes oesophageal squamous cell carcinoma motility and progression. J Pathol 215: 330-339, 2008

19. Lee MY, Chou CY, Tang MJ and Shen MR: Epithelialmesenchymal transition in cervical cancer: correlation with tumor progression, epidermal growth factor receptor overexpression, and snail up-regulation. Clin Cancer Res 14: 4743-4750, 2008.

20. Cervantes-Arias A, Pang LY and Argyle DJ: Epithelialmesenchymal transition as a fundamental mechanism underlying the cancer phenotype. Vet Comp Oncol 11: 169-184, 2013.

21. Chen H, Paradies NE, Fedor-Chaiken M and Brackenbury R: E-cadherin mediates adhesion and suppresses cell motility via distinct mechanisms. J Cell Sci 110: 345-356, 1997.

22. McInroy L and Määttä A: Down-regulation of vimentin expression inhibits carcinoma cell migration and adhesion. Biochem Biophys Res Commun 360: 109-114, 2007.

23. Vuoriluoto K, Haugen H, Kiviluoto S, et al: Vimentin regulates EMT induction by Slug and oncogenic $\mathrm{H}-\mathrm{R}$ as and migration by governing Axl expression in breast cancer. Oncogene 30: $1436-1448,2011$.

24. Karantza V: Keratins in health and cancer: more than mere epithelial cell markers. Oncogene 30: 127-138, 2011.

25. Di Como CJ, Urist MJ, Babayan I, Drobnjak M, Hedvat CV, Teruya-Feldstein J, Pohar K, Hoos A and Cordon-Cardo C: p63 expression profiles in human normal and tumor tissues. Clin Cancer Res 8: 494-501, 2002.

26. Lin DL, Whitney MC, Yao Z and Keller ET: Interleukin-6 induces androgen responsiveness in prostate cancer cells through up-regulation of androgen receptor expression. Clin Cancer Res 7: 1773-17781, 2001.

27. Xiao D and He J: Epithelial mesenchymal transition and lung cancer. J Thorac Dis 2: 154-159, 2010

28. Castrilli G, Tatone D, Diodoro MG, Rosini S, Piantelli M and Musiani P: Interleukin 1alpha and interleukin 6 promote the in vitro growth of both normal and neoplastic human cervical epithelial cells. Br J Cancer 75: 855-859, 1997.

29. Tjiong MY, van der Vange N, ten Kate FJ, Tjong-A-Hung SP ter Schegget J, Burger MP and Out TA: Increased IL-6 and IL-8 levels in cervicovaginal secretions of patients with cervical cancer. Gynecol Oncol 73: 285-291, 1999.

30. Cheng Y, Zhou Y, Jiang W, et al: Significance of E-cadherin, $\beta$-catenin, and vimentin expression as postoperative prognosis indicators in cervical squamous cell carcinoma. Human Pathol 43: 1213-1220, 2012 
31. Scanlon CS, Van Tubergen EA, Inglehart RC and D'Silva NJ: Biomarkers of epithelial-mesenchymal transition in squamous cell carcinoma. J Dent Res 92: 114-121, 2013.

32. Sullivan NJ, Sasser AK, Axel AE, Vesuna F, Raman V, Ramirez N, Oberyszyn TM and Hall BM: Interleukin-6 induces an epithelial-mesenchymal transition phenotype in human breast cancer cells. Oncogene 28: 2940-2947, 2009.

33. Bolós V, Peinado H, Pérez-Moreno MA, Fraga MF, Esteller M and Cano A: The transcription factor Slug represses E-cadherin expression and induces epithelial to mesenchymal transitions: a comparison with Snail and E47 repressors. J Cell Sci 116 : 499-511, 2003. 